\title{
Correction to: Optimizing component solution spaces for systems design
}

\section{Marco Daub $^{1}$ (D) Fabian Duddeck ${ }^{1,2} \cdot$ Markus Zimmermann $^{1}$}

Published online: 12 May 2020

(C) Springer-Verlag GmbH Germany, part of Springer Nature 2020

\section{Correction to: Struct Multidisc Optim \\ https://doi.org/10.1007/s00158-019-02456-}

8

The original version of this article unfortunately contains mistakes introduced during the publishing process. The mistakes and corrections are described in the following list:

1. The typographical error in the reference "Daub M (2017) Konvexe Optimierung am Beispiel volumenmaximaler einbeschriebener Rechtecksmengen. Master thesis, Universität Konstanz, Germany, http://nbn-resolving.de/ urn:nbn:de:bsz:352-0-420923" was removed.

2. The typeface of the following figures was adapted.

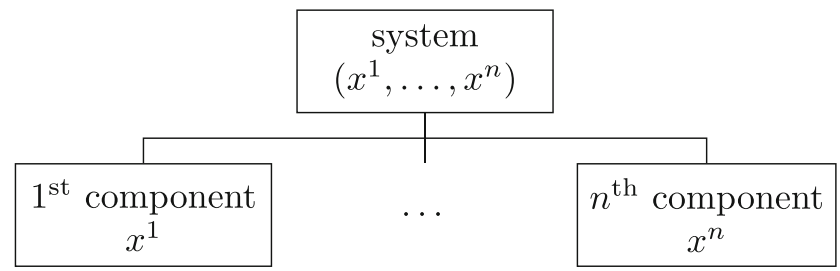

Fig. 1 A system composed of $n$ components each specified by a design variable vector $x^{k}, k=1, \ldots, n$

The online version of the original article can be found at https://oi.org/ 10.1007/s00158-019-02456-8

Marco Daub

marco.daub@tum.de

Fabian Duddeck

duddeck@tum.de

Markus Zimmermann

zimmermann@tum.de

1 Technische Universität München, Arcisstr. 21, 80333 München, Germany

2 Queen Mary University of London, Mile End Road, London E14NS, UK

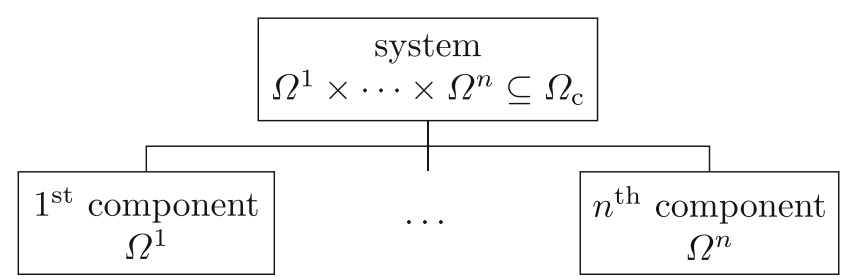

Fig. 2 Decomposition of the complete system solution space into component solution spaces

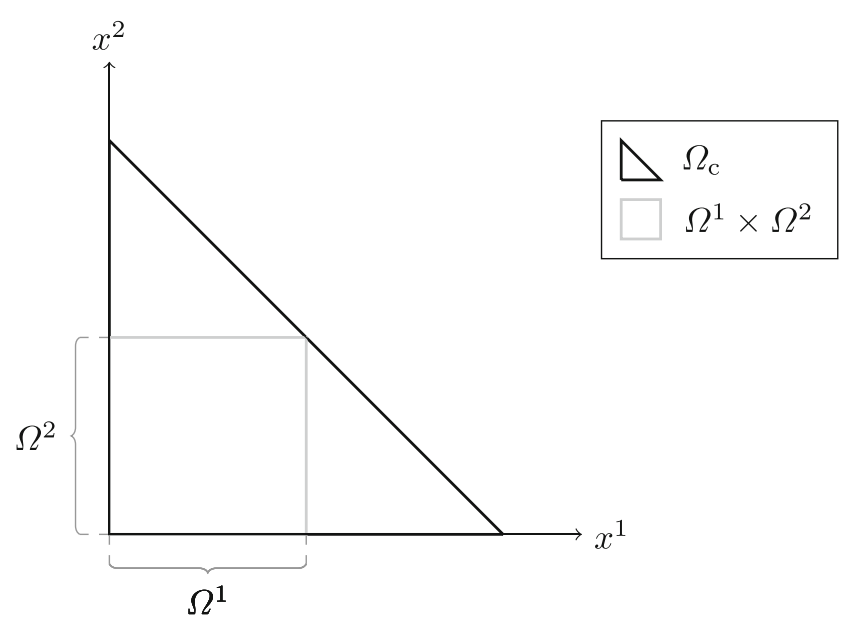

Fig. 3 Example of optimal component solution spaces $\Omega^{1}$ and $\Omega^{2}$ for a system composed of two components with one degree of freedom each

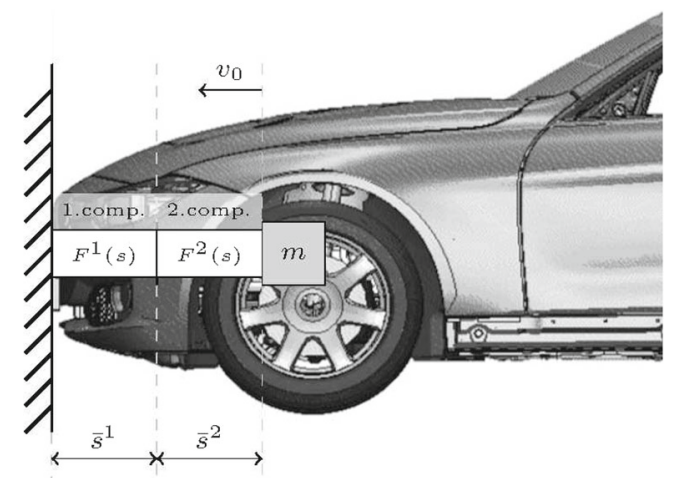

Fig. 4 Vehicle front structure for the simple crash design problem with two components and force-deformation characteristics $F^{1}(s), s \in[0 \mathrm{~mm}$, $\left.\bar{s}^{1}\right)$ and $F^{2}(s), s \in\left[0 \mathrm{~mm}, \bar{s}^{2}\right)$ 


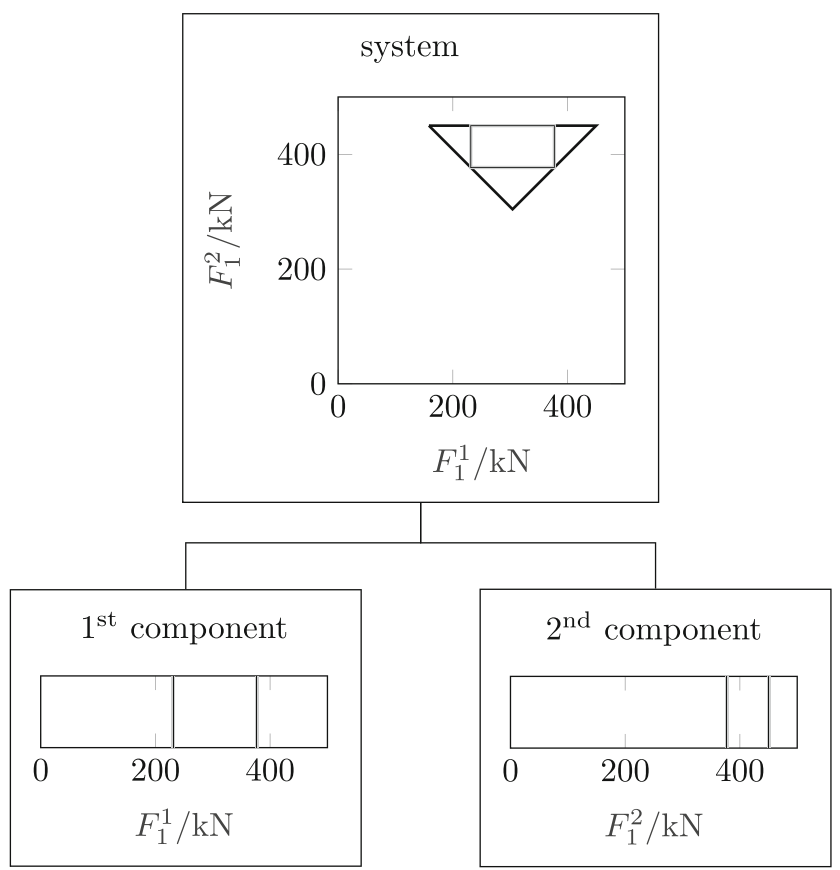

Fig. 5 Decomposition of the complete system solution space $\Omega_{\mathrm{c}}$ (thick black) for the simple crash design problem with $d^{k}=1$ into $\Omega_{\mathrm{bs}}^{1}, \Omega_{\mathrm{bs}}^{2}$ (thin black) and $\Omega_{\text {as }}^{1}, \Omega_{\text {as }}^{2}$ (gray)

Fig. 6 Decomposition of the complete system solution space $\Omega_{\mathrm{c}}$ for the simple crash design problem with $d^{k}=2$ into $\Omega_{\mathrm{bs}}^{1}, \Omega_{\mathrm{bs}}^{2}$ (black) and $\Omega_{\mathrm{as}}^{1}, \Omega_{\mathrm{as}}^{2}$ as (gray)

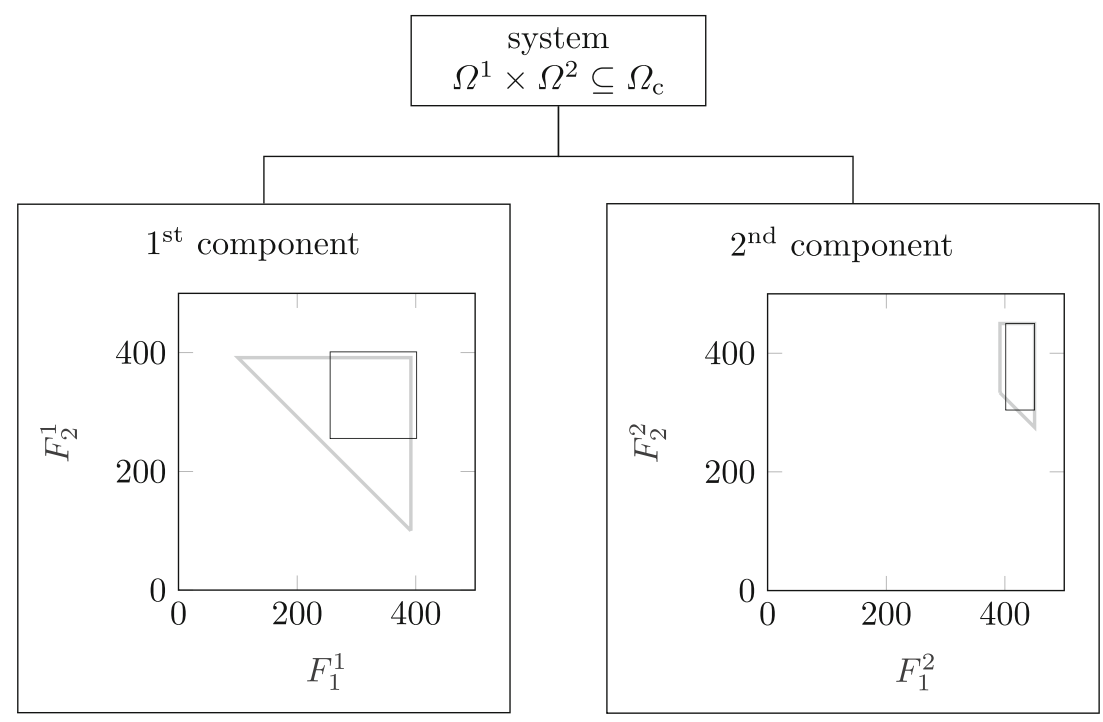


Fig. 7 Component solution spaces $\Omega^{k}$ and their corresponding region of possibly permissible characteristics both bounded by solid gray lines for different $d^{k}$. Inside, there are permissible (white dots) or non-permissible (gray dots) force-deformation characteristics. Outside, they are always non-permissible (black dots)

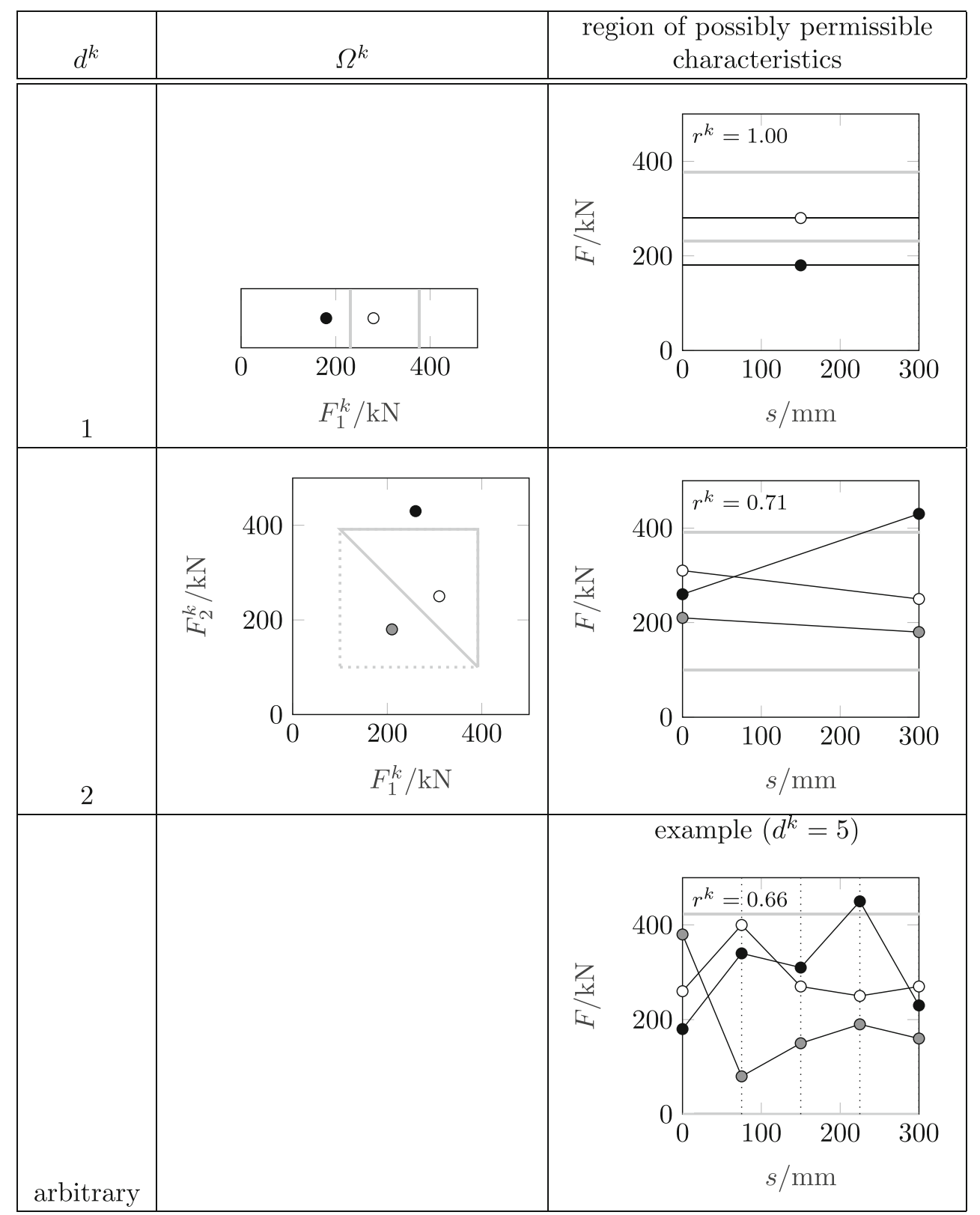


Fig. 8 Total volume (left) and average edge length (right) of box-shaped (bs) and arbitrarily shaped (as) component solution spaces
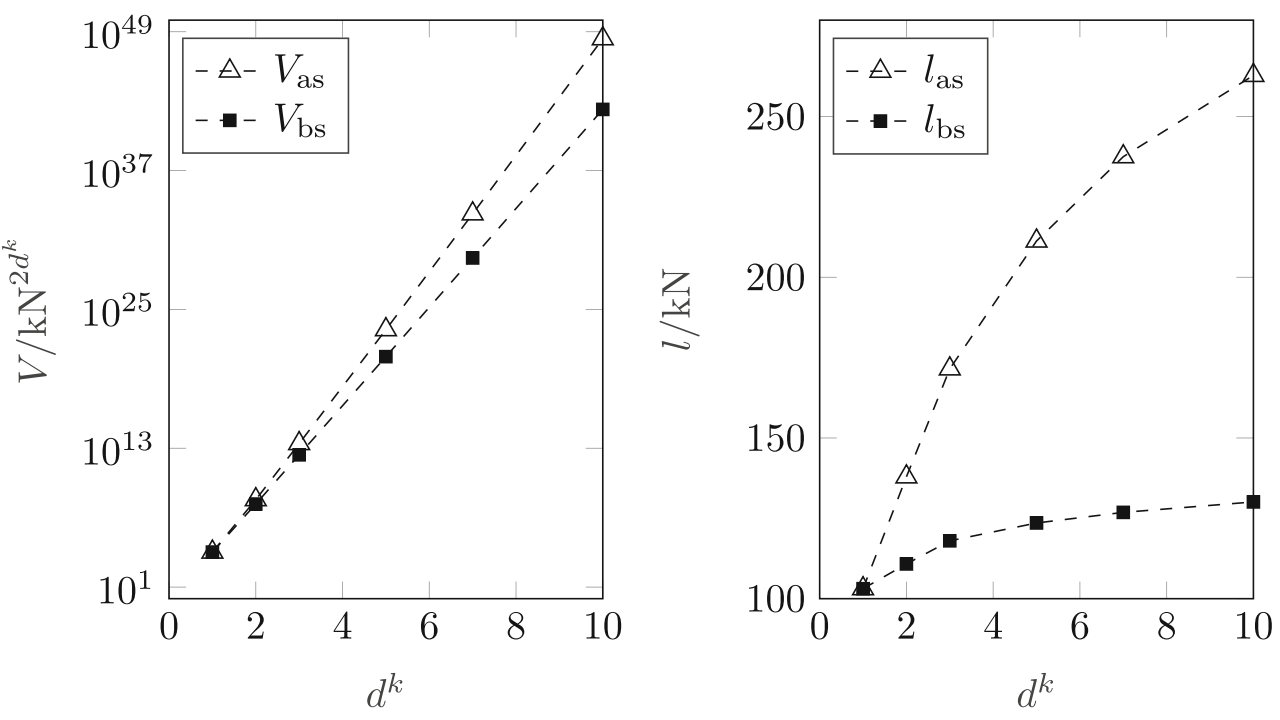

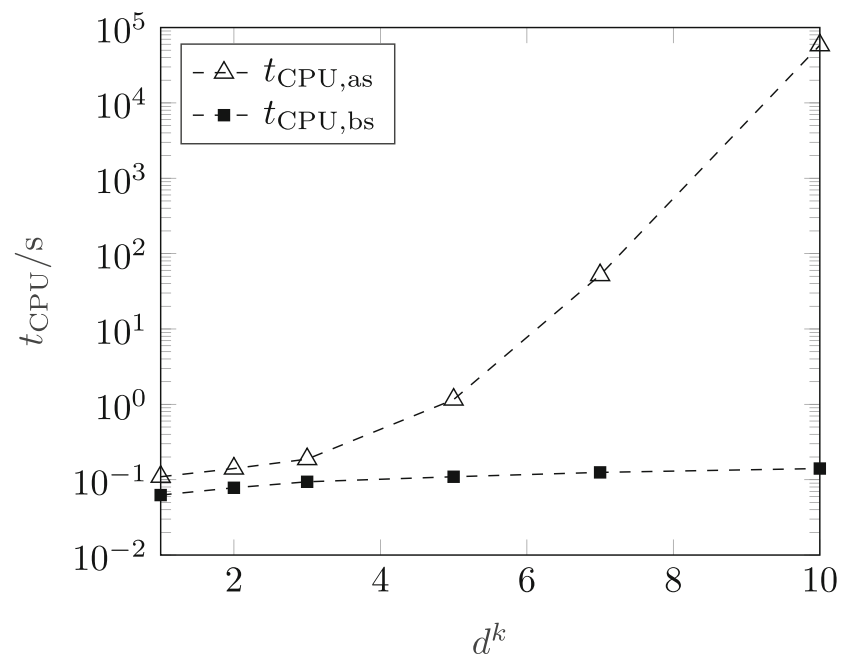

Fig. 9 CPU time for Intel(R) Xeon(R) CPU E5-1660 v4 @ $3.20 \mathrm{GHz}$ to calculate box-shaped (bs) and arbitrarily shaped (as) component solution spaces with analytically calculated volume 
Fig. 10 Vehicle front structure for the realistic crash design problem with eight components. The gray parts indicate deformable

structure and the black parts non-

deformable structure

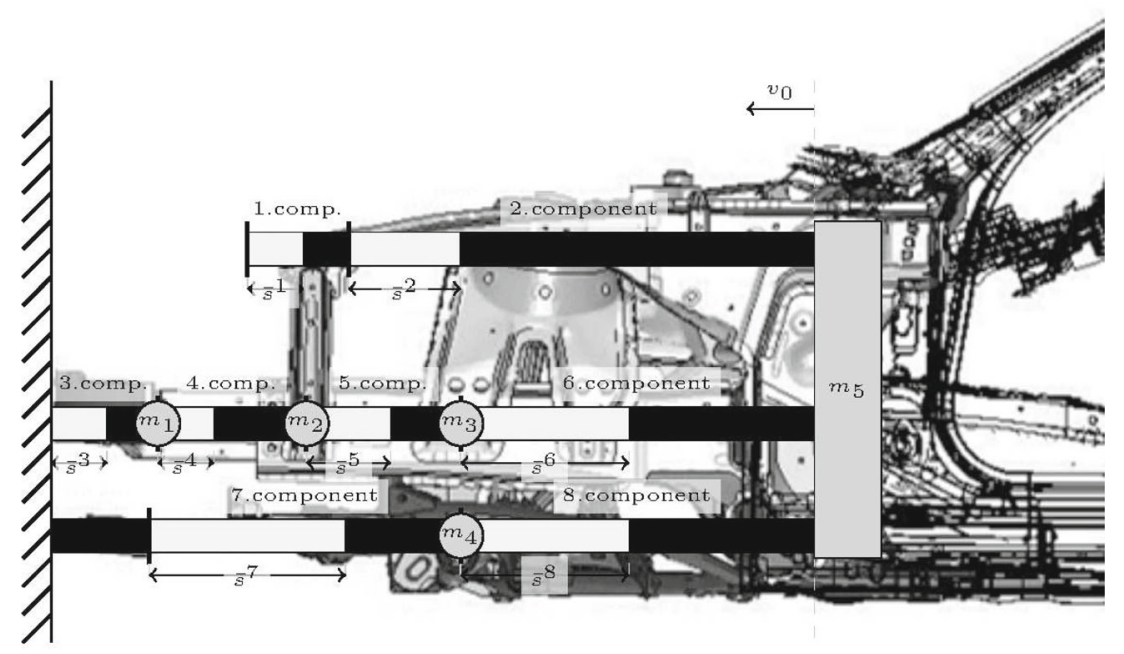

(a) Geometry space

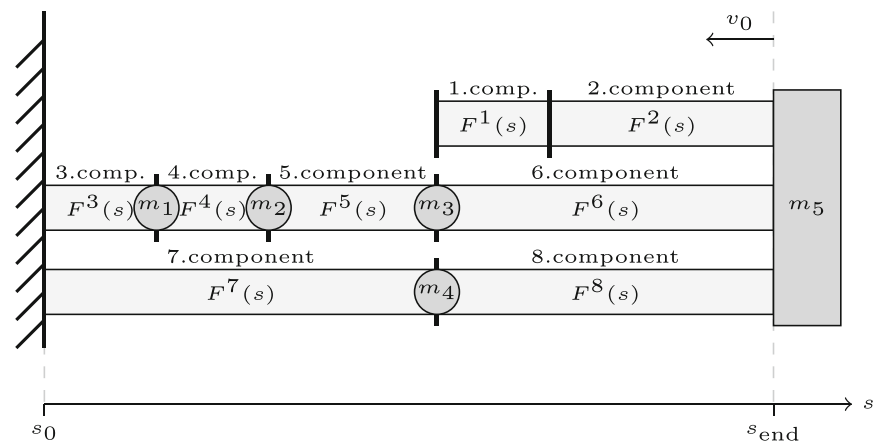

(b) Deformation space (stretched) 
Fig. 11 Regions of possibly permissible characteristics from optimal component solution spaces for optimal arbitrarily shaped component solution spaces (gray) and lower and upper bounds of the optimal box-shaped component solution spaces (black)

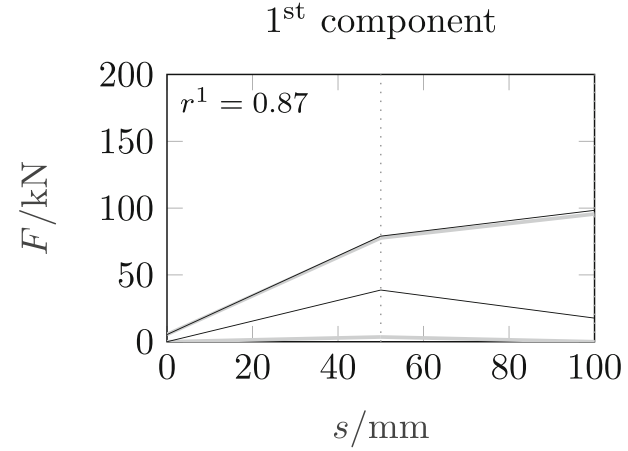

$3^{\text {rd }}$ component

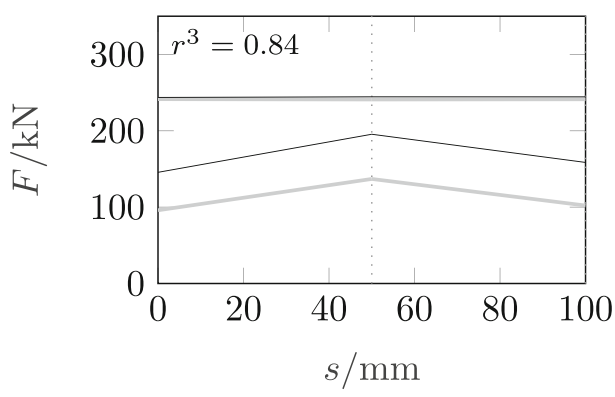

$5^{\text {th }}$ component

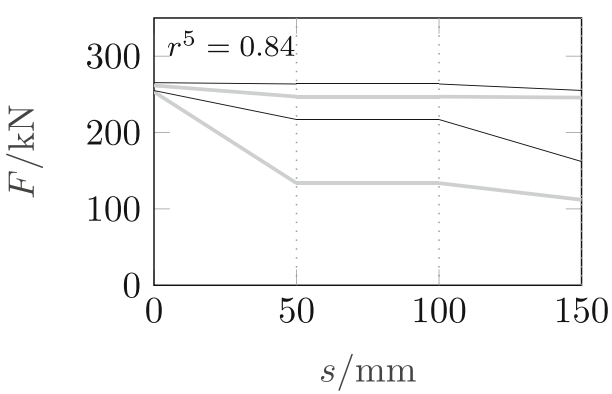

$7^{\text {th }}$ component

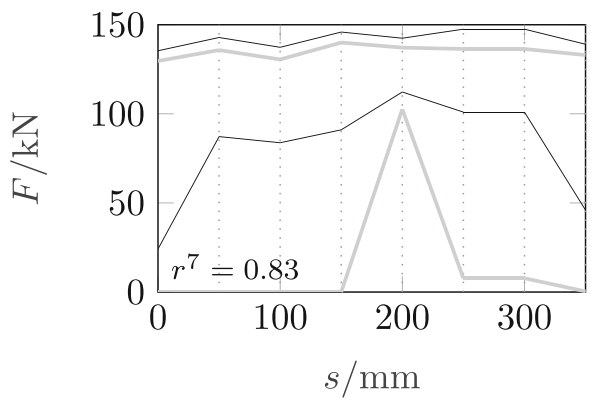

$2^{\text {nd }}$ component

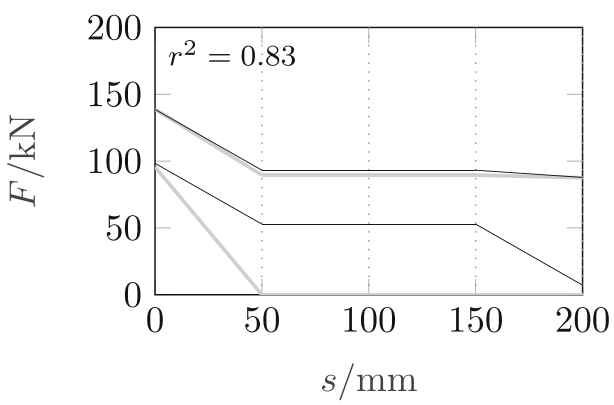

$4^{\text {th }}$ component

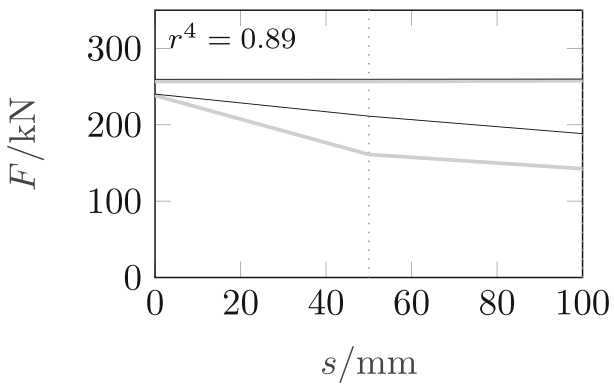

$6^{\text {th }}$ component

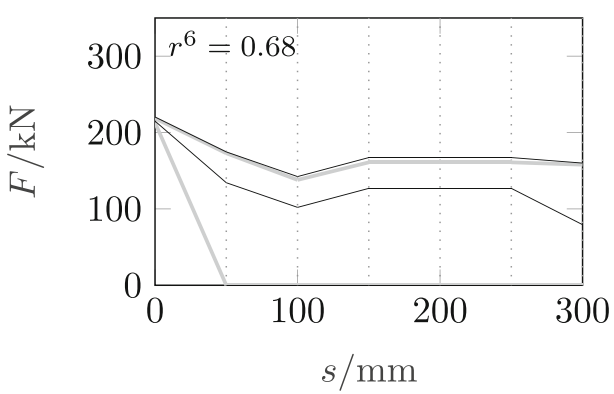

$8^{\text {th }}$ component

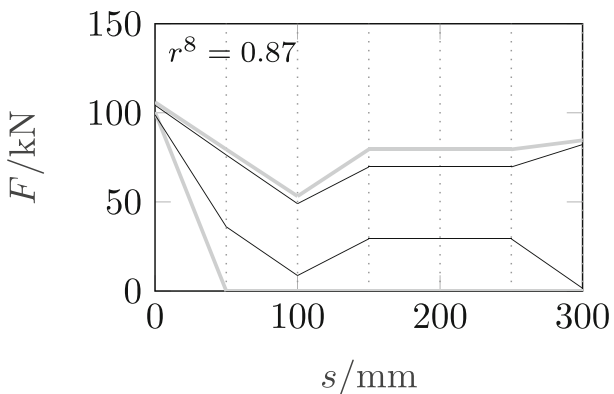

Publisher's note Springer Nature remains neutral with regard to jurisdictional claims in published maps and institutional affiliations. 